\title{
Atherosclerosis (Also Coronary Artery Disease), Rheumatoid Vasculitis, Morbus Alzheimer, Stroke Etc
}

\author{
Ferdynand Barbasiewicz* \\ University of Warsaw, Poland \\ Received: September 10, 2017; Published: September 21, 2017 \\ *Corresponding author: Ferdynand Barbasiewicz, Warsaw, University of Warsaw, Poland, Email: centrum@klawiterapia.com
}

\section{Opinion}

According to American research 3 million various sorts of molecular endogenous factors circulate in the blood of a healthy person, including around 100 thousand selective antibodies (established by me, I have evidence). Moreover, there are about 10 million submolecular neurochemical transmitters and neurotransmitters in blood. All molecular factors, antibodies and neurochemical submolecular transmitters are controlled by biocybernetic non-invasive stimulation with clavics or wellknown toothpicks through dermal receptors, collagen innervated fibres, numerous biologically active points, also cryogenic, acupuncture ones and the nervous system. The entire method of clavitherapy, which I have been investigating and experimenting with for over 50 years with verification in clinical conditions, is formulated methodically in 1100 algorithms with the network of actions quantified with medical diagnosis for various functional dysfunctions and diseases described and expressed pictorially in the books: Clavitherapy and the Atlas of Clavitherapy.

To characterize the method of clavitherapy most generally it is essential to recognize as art stimulation with clavics, like lat. clavus = nail in controlling dermovisceral repair processes of the molecularly disturbed endogenous homeostasis of the human body out of negligence and ischaemia. The other clinical achievement and even my discovery was the precise etiological specification of functional and structural disorders of the nervous system forming the main causes of many various drug-resistant ailments. In the 80s in Klinika Neurologiczna Szpitala Klinicznego MSW (Neurological Clinic of the Clinical Hospital of the Ministry of Internal Affairs) in Warsaw I developed an effective and simple method of liquidating in several days demyelinating disturbances in the brain and in various sorts of peripheral nervous fibres and neuron palsies with glioblastomas elicited by the earliest changes of the malignant neoplasm, which are entirely drug-resistant. The range of effectiveness of clavitherapy is expressed in short in the publication entitled Molecular medicine of the distant future available just today.
It also concerns resuscitation without the defibrillator and massaging the chest. Presently there are several masters of clavitherapy and there are two people admitted into a doctoral programme at medical universities. The fourth edition of Clavitherapy is prepared but I have no money for printing houses. Awaiting in the near future the successful incorporation of the method of clavitherapy into education, research and specialization programmes at medical universities, I kindly ask you to become acquainted with the stance of Polskie Towarzystwo Lekarskie (Polish Doctors' Society). Please read the attached publications and watch short videos at: ATVN on www.klawiterapia.com and the PTL board's opinion www.youtabe.com/wath?v=ymZOfeYLDtk, www. instytutklawiterapii.com/yt.

At the same time I inform that in the Centre of Clavitherapy Source I have many documents which form clinical proofs of my actions. Moreover, please enter Clavitherapy in English in Google, where you can spot how the molecular method of clavitherapy is disseminated around the world. I remain yours sincerely and give the best regards - Ferdynand Barbasiewicz, the author of the method of clavitherapy and e-book: 21st Century Eden (regards new cheap technically simple capabilities of remedying ecological and climatic negligence caused by the human), Email address: centrum@klawiterapia.com , telephone: 227298053.

I have resolved to respond to curiosity regarding research into atherosclerosis, also coronary artery disease, morbus alzheimer, leprosy. The problem of atherosclerosis is one of the most significant threats to life of many people worldwide. In the molecular method of biocybernetic clavitherapy I hold a very simple algorithm with the network of actions and the technique of a stimulation procedure, with which I activate in the muscularis of the blood vessels with deposits the idiosyncratic submolecular enzyme (some sort of protein), which literally in several days accurately clears the flow of blood in the blood vessels with a confirmation of the Doppler test. This idiosyncratic enzyme simultaneously disposes of any deposits so there is no risk of triggering an obstruction in another place. 
If you are interested in the molecular method of biocybernetic clavitherapy, not only the natural clearing of the flow in the blood vessels, prepare a few patients who have an exacerbated flow of blood, eg in 50 per cent due to atheromatous deposits, and my assistants and I in 10 days will clear the inside of the blood vessels with no risk for the patient's health. The procedures are very brief and are affected in 20-30 minutes but it is necessary to repeat the stimulation twice a day in a weekly therapy. The procedures can be performed by the person interested after being given simple instruction. After the first stimulation procedure for biologically active points a definitely better frame of mind will be felt and the vascular pain will be eliminated. It also concerns the pain of rheumatoid vasculitis and stimulation must be performed on the central surface of the arm on the biceps (biceps brach ii, point 12 on the meridian of the triple heater, which can be indicated to you by any doctor knowing acupuncture).

In the cure you can stimulate topically with two toothpicks the inside edge of the tibial bone from the knuckle of the upper ankle joint spot by spot as far as the knee on both limbs. Where you feel severe pain it is necessary to stimulate 2-3 seconds each until the pain abates. The other biologically active point is found on the outside edge of the sagittal bone about $5 \mathrm{~cm}$ above the outside knuckle of the ankle joint. You can effectively actuate the fibrin of the blood vessels when a tendency for varicose veins appears early. Stimulation must be led $2-3 \mathrm{~cm}$ from the axis of the inside knuckle towards the big toe, acupuncture point no. 5 on the meridian of the spleen and pancreas. The procedures must be repeated twice a day over a week along with clavitherapy exercises illustrated by the film on the site: www.instytutklawiterapii.com. Next apply a diet poor in pork in favour of rabbit, duck, goose, turkey, fish with a high content of well crushed raw vegetables with an addition of garlic, a small spoon of flax oil, sprinkled with lemon juice.

\section{Morbus Alzheimer, Therapeutic Procedure with the Molecular Method of Bio-Cybernetic Clavitherapy}

It is recommended to stimulate the whole surface of the skin on the head, face, cervix and neck and unblock the 4th chamber of the brainstem. There at the very bottom there is the peripheral vascular and motor centre, which, after it relieves, normalizes blood pressure, see the Atlas of Clavitherapy under the lobe of the external ear the nervous sympathetic trunk. Next according to the indications in the Atlas of Clavitherapy it is necessary to liquidate cortical tensions PaM-3 and central GRT-15. It is also necessary to clear the 5th cranial nerve Wż-20 and PaM-1 in the centre of the fontanelle bone of the cerebral centre responsible for the blood supply of the brain. After these actions of the 20 minute procedure perform non-invasive stimulation of acupuncture points to liquidate the Alzheimer disorder: Jg-11; Ż-8; ST-2; Jc-6, N-3; 0-6; Po-17 (Nervous Sympathetic Trunk); Wż-20; W-3; GRT: 15, 16, 20, 23; PaM-1, U: 25, 34, 51. The same procedural sequence must be performed twice a day over 10 days until the pain abates together with the above diet and clavitherapy exercises posted on the website: www.klawiterapia.com and www.instytutklawiterapii. com/yt.

\section{Disrupted EEG records}

Caution! If there is no pathology in the morphological structures of the brain (confirmed by MRI tests) and the peripheral nerves, and if there are disturbances against a psychosomatic, functional or palsied background of the nervous system, especially in the region of the brainstem and cranial nerves. After a clavitherapy procedure, removing cortical and central tensions and major fibres of the peripheral nerves controlling the vegetative system, incorrect EEG records withdraw efficiently and effectively. See the Atlas of Clavitherapy-detailed relief of the head, cervix, neck and back for removing nervous, psychosomatic disturbances. I remain your sincerely and give the best regards-Ferdynand Barbasiewicz, author of the Method of Clavitherapy and the E-Book 21-Century Eden.

\section{Leprosy}

Dear Sister, Cecylia Bachalska, please send the information below to Dr. Helena Pyż from the Jeevodaya Centre in India.

\section{Dear Sister,}

It is worth knowing that the aetiology of leprosy is closely related to the demyelinating neuropathy of the nervous vegetative fibres, and also the nervous fibres controlling the dynamics of the muscularis of the blood vessels or with some ischaemic obstruction. An attempt to clarify it deductively: if in a specific morphological structure or in any organ efficient blood supply is disabled, white algo-tangential and algo-dystrophic blotches appear in the dermatome on the skin. When the ambient temperature over the whole day exceeds the standard temperature of a healthy person and more degrees and there is no good blood supply, oxygenation, feeding with fresh blood rich in nutritional values with molecular endogenous factors of the specific morphological structure. There is no cooling of blood circulation with the liver. Then at a very high outside temperature over the whole day over a few months due to demyelinating neuropathy on the sympathetic (parasympathetic) fibres controlling the dynamics of the muscularis of the blood vessels or nervous vegetative fibres a total enzymatic and metabolic collapse occurs and the cells and tissues undergo rapid decomposition and fall off the healthy tissues with good blood supply in a specific anatomic and morphological zone.

In the Old Testament there are descriptions of the procedure of priests in case white blotches appear on the surface of the skin of a give person. In the first period small white blotches appear (do not confuse it with albinism) on the human skin, the priest isolated the person with small blotches periodically and over some time observed whether the condition of the tissues underwent deterioration or not. If, for example after 2-3 weeks, the tissues on the skin did not undergo decomposition, he released them from observation and declared that they were pure and harmless for the milieu. A conclusion can be drawn from this diagnosis that algotangential or algo-dystrophic blotches in this person, who had been declared pure, emerged on the pyramidal, motor nervous fibres and then they could have trouble with motor functions or the dynamics of skeletal muscles, but the body tissues did not decompose and 
the flesh did not fall off. Whereas when the priest having done observation noticed that the tissues undergo decomposition and the flesh begins to fall off, he declared that that person was impure and referred him for good till the end of life to a carefully isolated lazaretto.

A subjective conclusion of the author of clavitherapy: the person suffered demyelinating neuropathy on the vegetative or sympathetic and parasympathetic fibre regulating the inside of the vessel and the dynamics of the muscularis of the blood vessels or complete ischaemia occurred due to an obstruction, local ischaemic stroke or long-lasting malnutrition at a high temperature of the climate. A trauma with infection distinctly causes blood circulation to cease in a specific morphological structure. Complete lack of blood circulation triggered malnutrition, a shortage of oxygen and a shortage of blood cooling in the liver. Lack of nutrition, cooling the cells and tissues in a longer time, in a specific morphological zone, in a very high ambient temperature round the clock exacerbated the symptoms of leprosy. The cause affected a complete end of the enzymatic and metabolic functions at subcellular level of a specific morphological, anatomic structure of a specific endogenous level, and then the flesh undergoes atrophy, decomposition and can fall off. If the above-described demyelinating neuropathy occurs in our climatically moderate latitude, a person can live over a longer time and the flesh does not fall off. It tends to end up with a non-malignant neoplasm. It can be the case that the cause of leprosy is the permanent malnutrition of the human body and the disappearance, decrease in the level of a specific sort of endogenous factors. Whereas, claiming that it is a viral, contagious disease is not fully warranted as viruses can manifest themselves, develop only at the stage of leprosy development due to the locally idiosyncratic attenuated immunity. I remind you that the above interpretation of the aetiology of leprosy is a subjective deductive assessment of the author of the method of clavitherapy - it requires more accurate clinical tests. It is worth noting that there are people, volunteers, who on a daily basis in their time tended to those sick with leprosy and do not get infected, eg the late St Mother Teresa of Calcutta.

"Caring about the conditions of existence in line with the universal wisdom of Nature, let us respect any gift of life and let us be happy also with others "happiness" the author's lifetime motto.

I remain Yours Sincerely and give the best regards - Ferdynand Barbasiewicz, author of the Molecular Method in Biocybernetic Clavitherapy and E-Book 21st Century Eden. PS-For more information see the website: www.klawiterapia.com

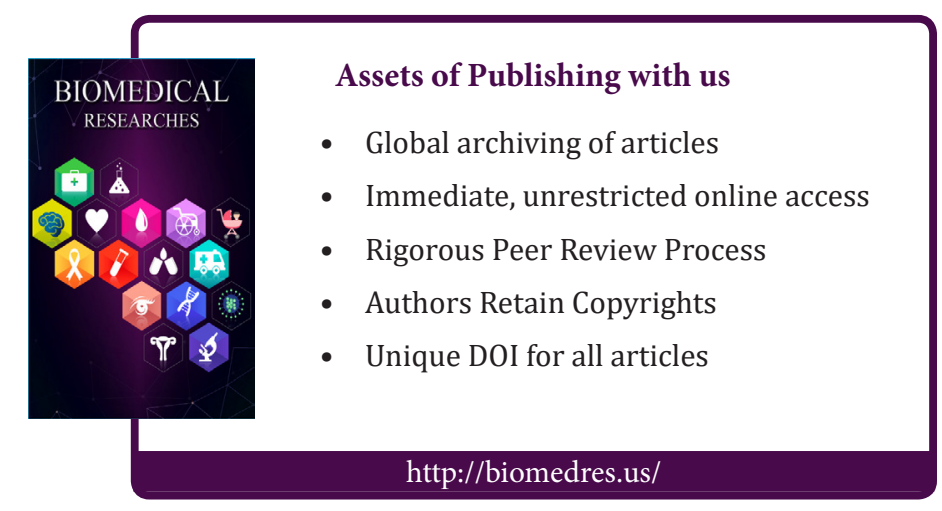

